\title{
Development of genetic maps for grapevine hybrid populations (Vitis vinifera L. $\times$ Muscadinia rotundifolia Michx.)
}

\author{
Lytkin K. ${ }^{1 *}$, Grigoreva E. ${ }^{1,2,3}$, Karzhaev D. ${ }^{1}$, Volkov V. ${ }^{1,3}$, Potokina E. ${ }^{1,3}$ \\ ${ }^{1}$ Saint Petersburg State Forest Technical University, Saint Petersburg, Russia \\ ${ }^{2}$ Saint Petersburg State University, Saint Petersburg, Russia \\ ${ }^{3}$ All-Russian National Research Institute of Viticulture and Winemaking 'Magarach', RAS, Yalta, Russia \\ *L.Grigoreva@gmail.com
}

Key words: genetic map, vitis, hight-throughput genotyping

Motivation and Aim: Resistance to mildew is an important agrobiological trait for winegrowers. However, most cultivated grapevine varieties do not carry resistance genes, while their wild relatives have such genes. The most promising solution is interspecific crosses between cultivated and wild species of Vitis. The North American species of Vitis rotundifolia is a good source of grapevine resistance to pathogens and pests. However, the problem of interspecific crosses within Vitis is the different diploid set of chromosomes $(2 n=38$ for $V$. vinifera and $2 n=40$ for $V$. rotundifolia $)$. Despite this, as a result of several successful attempts, some interspecific hybrids were obtained. One of them is the NC6-15 hybrid that was used as a resistant parent for pseudobackcrosses with $V$. vinifera varieties. Our goal is to construct a genetic map of the hybrids and recover their progeny to see how resistance loci are inherited. For this goal three interspecific crosses were investigated.

Results: We generated genetic maps for different interspecific crosses using female genotype M.N.31-77-10 ('Nimrang' × 'Seibel 13666') and two male parental genotypes 2000-305-143 and 2000-305-163. In total 139 progeny genotypes $(66+43+30)$ and parental genotypes (female M. No. 31-77-10; male 2000-305-143, 2000-305-163) were genotyped using the double-digest RADseq approach. In total, 309,762,340 high-quality reads were obtained. For the female parent, which was common for all of the hybrid populations, 6,717,571 reads were generated; for male parents 2000-305-143 and 2000$305-163,4,414,422$ and 9,010,505 reads were obtained, respectively. The progeny of population 2-11 provided 140,445,297 reads, the progeny of population 3-11 provided $97,219,515$ reads, and the progeny of population 4-11 provided 51,955,030 reads. Reads were aligned against reference genomes ( $V$. vinifera, $V$. rotundifolia), SNP calling was performed using different pipelines: Stacks, GATK, Tassel5 and genetic maps were constructed using rqt12 software.

Conclusion: The genetic maps were constructed for three hybrid populations. This allows to investigate heritability of resistant loci in this populations.

Acknowledgements: The research was funded by the Russian Science Foundation (project No. 20-16-00060). 